\title{
The expression of CD23 in cutaneous non-lymphoid neoplasms
}

Background: Cluster designation 23 (CD23) is generally used as a lymphoid marker. Its utility in cutaneous epithelial tumors has never been studied. In our routine practice, we observed that CD23 reacted strongly with eccrine and apocrine secretory coils.

Methods: Immunohistochemical staining of CD23 was performed in a total of 131 cases of apocrine, eccrine, follicular and other cutaneous non-lymphoid tumors.

Results: CD23 expression was detected in all benign apocrine tumors and in half of benign eccrine tumors, particularly those derived from secretory coils. CD23 staining was seen in 42\% (8/19) of microcystic adnexal carcinoma (MAC), while no staining was observed in tumor cells of desmoplastic trichoepithelioma, morpheaform basal cell carcinoma and syringoma. All mammary and extramammary Paget's disease were labeled with CD23. In comparison, pagetoid Bowen's disease, melanoma in situ and sebaceous carcinoma exhibited negative staining. In addition, CD23 reacted diffusely with cutaneous mucinous eccrine carcinoma in a manner similar to breast or colonic adenocarcinoma.

Conclusion: CD23 appears to be a reliable immunohistochemical marker of the eccrine/apocrine secretory coil and helpful in identifying sweat gland tumors of such origin. It is of ancillary value in

differentiating MAC from its mimicker. CD23 is a useful addition to the diagnostic immunohistochemical panels for Paget's disease.

Carvalho J, Fullen D, Lowe L, Su L, Ma L. The expression of CD23 in cutaneous non-lymphoid neoplasms.

J Cutan Pathol 2007; 34: 693-698. (C) Blackwell Munksgaard 2006.
Jason Carvalho', Douglas

Fullen $^{1,2}$, Lori Lowe ${ }^{1,2}$, Lyndon

$\mathrm{Su}^{1,2}$ and Linglei $\mathrm{Ma}^{1,2}$

${ }^{1}$ Department of Pathology and

${ }^{2}$ Department of Dermatology, University of

Michigan Medical Center, Ann Arbor, MI, USA
Linglei Ma, Department of Pathology,

Dermatopathology Division,University of Michigan, M3260, Medical Sciences 1, 1301 Catherine Road, Ann Arbor, Ml 48109-0602, USA

Tel: +17347644579

Fax: +17347644690

e-mail: lingleim@umich.edu

Accepted for publication September 15, 2006
Cluster designation 23 (CD23) is a type II transmembrane glycoprotein, which is the low-affinity receptor for IgE. It is expressed by $\mathrm{B}$ cells, subsets of monocytes/macrophages, T cells and eosinophils. ${ }^{1,2}$ Although GD23 is widely used as a lymphoid marker $^{3}$ in diagnosing chronic lymphocytic leukemia, its expression has been demonstrated in normal intestinal epithelium by several groups. ${ }^{4-6}$ Previously, a few hematopoietic markers were found to be expressed in some non-hematopoietic tumors, such as CD56 in small-cell carcinoma, CD10 in renal cell carcinoma ${ }^{8}$ and CD5 in thymic carcinoma, breast and gastrointestinal adenocarcinoma. ${ }^{9-11}$ However, at present, there have been no studies evaluating the utility of CD23 in non-lymphoid tumors.

Cutaneous adnexal neoplasms comprise a heterogeneous group of tumors with eccrine, apocrine, follicular and sebaceous differentiation. Some of them share overlapping features and may present diagnostic challenges. A number of immunohistochemical markers ${ }^{12-16}$ have been used to determine the histogenesis of sweat gland tumors in an attempt to improve their identification and classification. CD44 was shown to react with the non-luminal surface of the normal eccrine secretory coil, but failed to act as a useful marker for specific forms of sweat gland differetiation. ${ }^{12}$ Staining with carcinoembryonic antigen (CCEA) has been shown in eccrine/apocrine ducts and in some eccrine secretory coils. ${ }^{13}$ Later on, IKH-4 was reported to stain eccrine secretory coil and tumors derived from it, proving its usefulness in differentiating eccrine from apocrine tumors. ${ }^{14}$ Recently, CD5 was detected in the eccrine/apocrine secretory coil, some sweat 


\section{Carvalho et al.}

gland tumors and Paget's disease. ${ }^{16}$ Despite the above effort, the histogenesis and classification of sweat gland tumors remains a subject of discussion.

In our routine evaluation of cutaneous lymphoid lesions, we observed that GD23 reacted strongly with certain non-lymphoid tissue, such as eccrine and apocrine secretory coils. We proposed that CD23 could be used to study the histogenesis of sweat gland tumors. In addition, we sought to determine whether CD23 could help differentiate microcystic adnexal carcinoma (MAC) from other cutaneous infiltrating tumors and/or separate Paget's disease from other pagetoid lesions. These aims prompted us to examine the CD23 expression in a large group of skin tumors of different lineages, as well as some non-cutaneous tumors.

\section{Methods}

After approval from the University of Michigan Institutional Review Board for human subject research, a search of the laboratory files of the University of Michigan Pathology Department from January 1995 to May 2006 was performed. From this search, we identified 131 cutaneous tumors, including 66 various benign cutaneous eccrine/ apocrine tumors, 19 MAC, 17 Paget's disease (mammary or extramammary), 2 mucinous eccrine carcinoma and 27 other cutaneous neoplasms of epithelial, follicular, sebaceous or melanocytic origin. In addition, nine cases of non-cutaneous adenocarcinoma (breast or colon) were also included in this study. All tumors examined are listed in Table 1.
The original diagnoses were confirmed by histologic review (L. M.). Using a mouse anti-human monoclonal CD23 antibody at a 1:10 dilution (clone BU38; The Binding Site, San Diego, CA, USA), immunohistochemical staining was performed on $5-\mu \mathrm{m}$ sections prepared from the formalin-fixed and paraffin-embedded tissue blocks. All slides were pretreated with protease for $16 \mathrm{~min}$. Staining was performed on a Ventana Benchmark XT system (Ventana Medical Systems, Tucson, AZ, USA) using the iVIEW diaminobenzidine reaction kit for visualization.

The staining pattern and intensity of CD23 were assessed on each stained slide. Any cytoplasmic staining was considered positive. The percentage of positive lesional cells was recorded as one of the four categories: $>50 \%,>10-50 \%, 1-10 \%$ and $<1 \%$. Cases classified as $<1 \%$ staining were those stained with rare single cell, which were not tumor cells. The intensity of staining was graded as strong or weak.

\section{Results}

In normal skin, CD23 labeled the luminal cells of the eccrine/apocrine secretory coils (deep portion of the glands) but not the outer myoepithelial cells (Fig. 1A, B). The eccrine/apocrine intradermal and intraepidermal ducts, follicular structures and sebaceous glands were not stained. Occasionally, the dendritic processes of some dermal dendrocytes demonstrated weak staining. In addition, CD23 staining was noted in salivary glands, normal breast tissue (lobules and lactiferous ducts) as well as colonic epithelium.

Table 1. CD23 immunostaining in cutaneous and non-cutaneous neoplasms

\begin{tabular}{|c|c|c|c|c|c|c|c|c|c|}
\hline \multirow[b]{2}{*}{ Tumor origin } & \multirow[b]{2}{*}{ Tumor type } & \multirow[b]{2}{*}{$\mathrm{n}$} & \multicolumn{5}{|c|}{ Percentage of CD23-positive cells } & \multicolumn{2}{|c|}{ Staining intensity } \\
\hline & & & $>50$ & $>10-50$ & $1-10$ & $<1^{*}$ & 0 & Strong & Weak \\
\hline \multirow{11}{*}{ Apocrine/eccrine } & Hidradenoma & 12 & 1 & 2 & 6 & 0 & 3 & 5 & 4 \\
\hline & Hidrocystoma & 7 & 7 & 0 & 0 & 0 & 0 & 7 & 0 \\
\hline & Syringocystadenoma papilliferum & 5 & 5 & 0 & 0 & 0 & 0 & 5 & 0 \\
\hline & Hidradenoma papilliferum & 6 & 6 & 0 & 0 & 0 & 0 & 6 & 0 \\
\hline & Poroma & 9 & 0 & 0 & 2 & 0 & 7 & 2 & 0 \\
\hline & Syringoma & 6 & 0 & 0 & 0 & 0 & 6 & 0 & 0 \\
\hline & Eccrine spiradenoma & 8 & 0 & 4 & 4 & 0 & 0 & 4 & 4 \\
\hline & Cylindroma & 7 & 0 & 0 & 7 & 0 & 0 & 6 & 1 \\
\hline & Chondroid syringoma & 6 & 2 & 3 & 1 & 0 & 0 & 5 & 1 \\
\hline & Microcystic adnexal carcinoma & 19 & 3 & 1 & 4 & 0 & 11 & 5 & 3 \\
\hline & Mucinous eccrine carcinoma & 2 & 2 & 0 & 0 & 0 & 0 & 2 & 0 \\
\hline Epithelial & Morpheaform basal cell carcinoma & 6 & 0 & 0 & 0 & 1 & 5 & 1 & 0 \\
\hline Follicular & Desmoplastic trichoepithelioma & 7 & 0 & 0 & 0 & 3 & 4 & 3 & 0 \\
\hline \multirow[t]{4}{*}{ Pagetoid } & Mammary Paget's disease & 8 & 8 & 0 & 0 & 0 & 0 & 8 & 0 \\
\hline & Extramammary Paget's disease & 9 & 9 & 0 & 0 & 0 & 0 & 9 & 0 \\
\hline & Pagetoid Bowen's disease & 6 & 0 & 0 & 0 & 0 & 6 & 0 & 0 \\
\hline & Melanoma in situ & 5 & 0 & 0 & 0 & 2 & 3 & 0 & 2 \\
\hline Sebaceous & Sebaceous carcinoma & 3 & 0 & 0 & 0 & 0 & 3 & 0 & 0 \\
\hline \multirow{2}{*}{ Non-cutaneous } & Breast ductal adenocarcinoma & 4 & 4 & 0 & 0 & 0 & 0 & 4 & 0 \\
\hline & Colonic adenocarcinoma & 5 & 5 & 0 & 0 & 0 & 0 & 5 & 0 \\
\hline
\end{tabular}

*Cases classified as $<1 \%$ staining are those with rare single cell labeled. These cells do not appear to be the tumor cells. 
Fig. 1. The expression of CD23 in normal skin appendages. CD23 intensely labels the luminal cells of eccrine (A) and apocrine (B) secretory coils. However, the intradermal and intraepidermal ductal units (A) are negative $(\times 200)$.
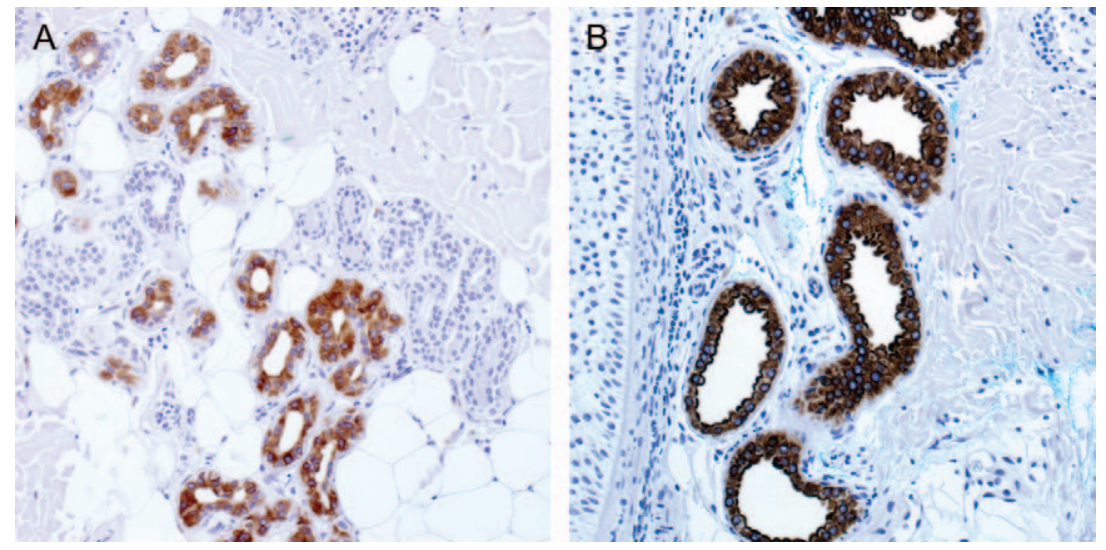

The results of staining are summarized in Table 1. A comparison was made between apocrine tumors and eccrine tumors (tumors which lack or show very focal apocrine differentiation). Apocrine tumors, such as hidradenoma papilliferum (6/6) and syringocystadenoma papilliferum (5/5), demonstrated diffuse $(>50 \%)$ and intense staining with CD23 (Fig. 2A). Likewise, all seven cases of eccrine/ apocrine hidrocystomas and one apocrine hidradenoma displayed similar staining pattern (Fig. 2B).

A variable CD23 staining pattern was noted in eccrine tumors. Most poromas (7/9) exhibited no staining (Fig. 2G). In two poromas, very focal staining was seen in an area of apocrine glandular differentiation. Nearly all cases of hidradenomas (11/12) demonstrated foci of luminal formation without large area of apocrine differentiation (i.e. apocrine decapitation, abundant eosinophilic cytoplasm) and were considered as eccrine hidradenomas. Of note, four of these demonstrated a focal apocrine component that was positive for GD23. Two others (without obvious apocrine differentiation) showed focal staining. Clear cell regions of hidradenoma were uniformly negative. As for eccrine spiradenoma (8/8), cylindroma $(7 / 7)$ and chondroid syringoma (6/6), CD23 focally labeled
Fig. 2. Diffuse and strong CD23 staining is observed in hidradenoma papilliferum (A) and hidrocystoma (B). Most eccrine poroma $(\mathrm{C})$ shows no staining. In contrast, focal luminal staining is present in chondroid syringoma $(D)(\times 200)$.
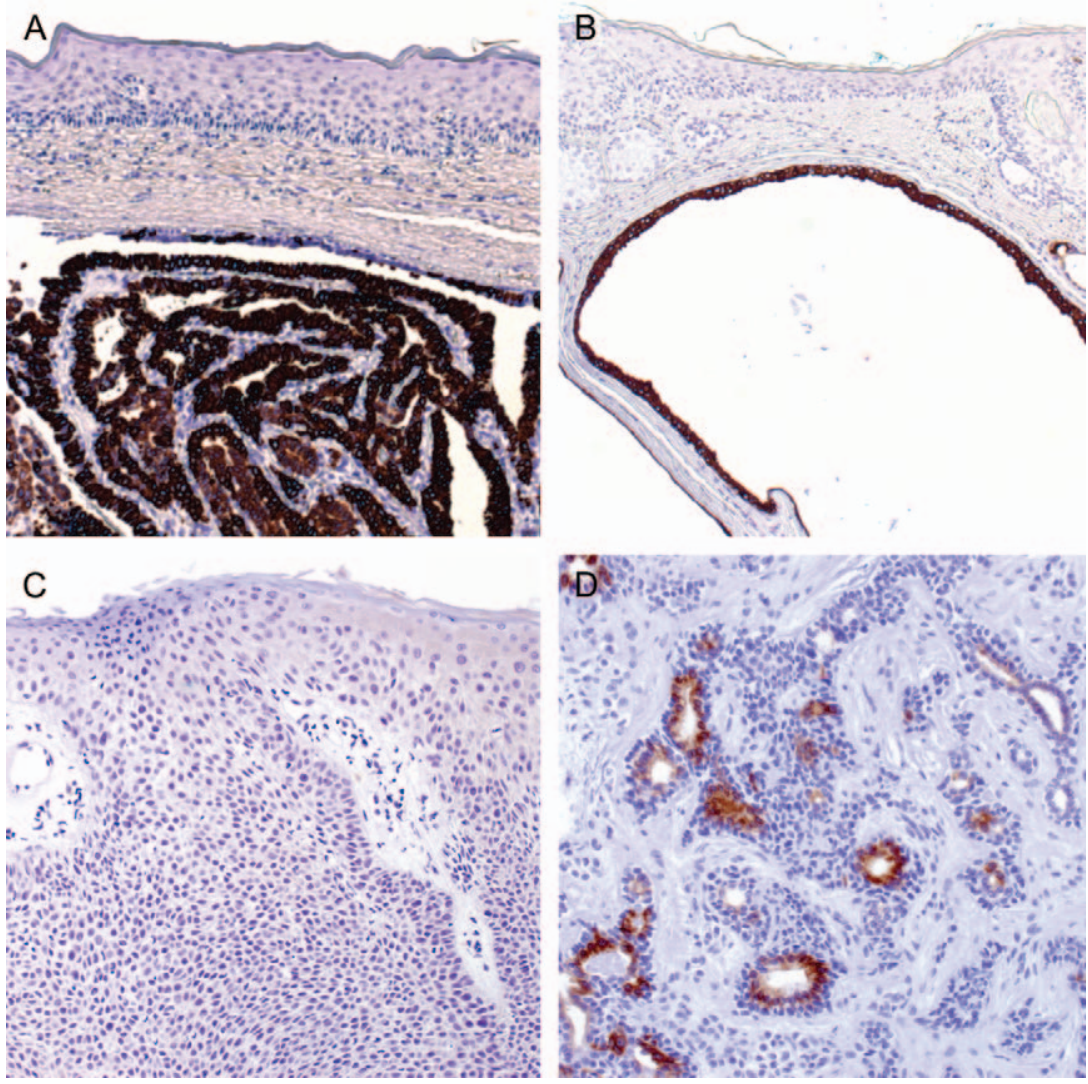


\section{Carvalho et al.}

the inner epithelial cells or glandular structures, sparing the peripheral lining cells (Fig. 2D). All mucinous eccrine carcinoma (2/2) showed diffuse and intense staining, a pattern similar to apocrine tumors.

Among 19 MAC, CD23 reacted diffusely or focally to eight cases $(42 \%)$, selectively targeting the glandular components (Fig. 3A). Areas of squamous or follicular differentiation and syringoid MAC were negative. The tumor cells of morpheaform basal cell carcinoma, desmoplastic trichoepithelioma and syringoma showed no staining (Fig. 3B). However, in some cases (Table 1), very rare single CD23positive cells, some of which showed dendritic morphology, were present within the tumor aggregates or the overlying epidermis and interpreted to not represent tumor cells. Similar cells were occasionally seen in normal epidermis (data not shown).

As illustrated in Fig. 4A, the tumor cells in both mammary (8/8) and extramammary (9/9) Paget's disease were highlighted strongly and diffusely by CD23, with membranous accentuation. In comparison, none of the pagetoid Bowen's disease $(0 / 6)$ or sebaceous carcinoma $(0 / 3)$ exhibited any reactivity (Fig. 4B). Of note, in two cases of melanoma in situ, a few dendrites in the epidermis were weakly stained by CD23.

As observed in cutaneous mucinous eccrine carcinoma, mucinous carcinoma of breast $(1 / 1)$ or colonic (3/3) origin was also decorated by CD23 in a similar pattern (Fig. 5A). Furthermore, diffuse and strong staining was observed in non-mucinous breast (3/3) and colon (2/2) adenocarcinoma (3/3) (Fig. 5B).

\section{Discussion}

In the current study, we characterized the staining pattern of CD23 in normal eccrine and apocrine glands and breast tissue. A distinction between the ductal and secretary portion of the sweat glands can be made by CD23 staining. In accordance with the previous studies, ${ }^{4-6}$ we observed CD23 expression in normal colonic epithelium.

Our data showed that CD23 is widely expressed in a variety of sweat gland tumors. We suspect that this expression may be dependent on the tumor type (eccrine or apocrine) and differentiation (ductal or secretory). As observed in the CD5 study, ${ }^{16}$ we found that all apocrine tumors expressed CD23 diffusely whereas only about half of the eccrine tumors demonstrated focal staining. Apocrine tumors (traditionally shown to have apocrine decapitation and ample amounts of eosinophilic cytoplasm) show strong CD23 reactivity, mimicking the staining pattern of normal apocrine secretory coils. Conventionally, some eccrine tumors (i.e. eccrine spiradenoma, cylindroma and chondroid syringoma) are believed to be derived from the eccrine secretory coil or the transitional region between duct and secretory coil. ${ }^{17,18}$ This origin is reflected in the staining pattern observed as tumors with focal luminal staining, a pattern analogous to normal eccrine secretory coil. In contrast, those eccrine tumors that possibly originate from ductal segments, ${ }^{19,20}$ such as poroma and syringoma, exhibited no staining, a pattern similar to that seen in the normal eccrine duct. Of interest, mucinous eccrine carcinoma, which is traditionally thought to be derived from eccrine secretory coil, ${ }^{21,22}$ showed a diffuse and strong staining pattern similar to that seen with clear-cut apocrine tumors. This makes one speculate as to whether or not mucinous eccrine carcinoma is indeed of eccrine origin and awaits further clarification.

Similarly, the origin of the tumor cells probably contributed to the variable CD23 staining seen in hidradenomas. We suspect that those with overlapping features of poroma may be derived from the upper portion of the sweat gland and showed no staining. Hidradenomas with extensive clear cell regions were not labeled possibly because these cells
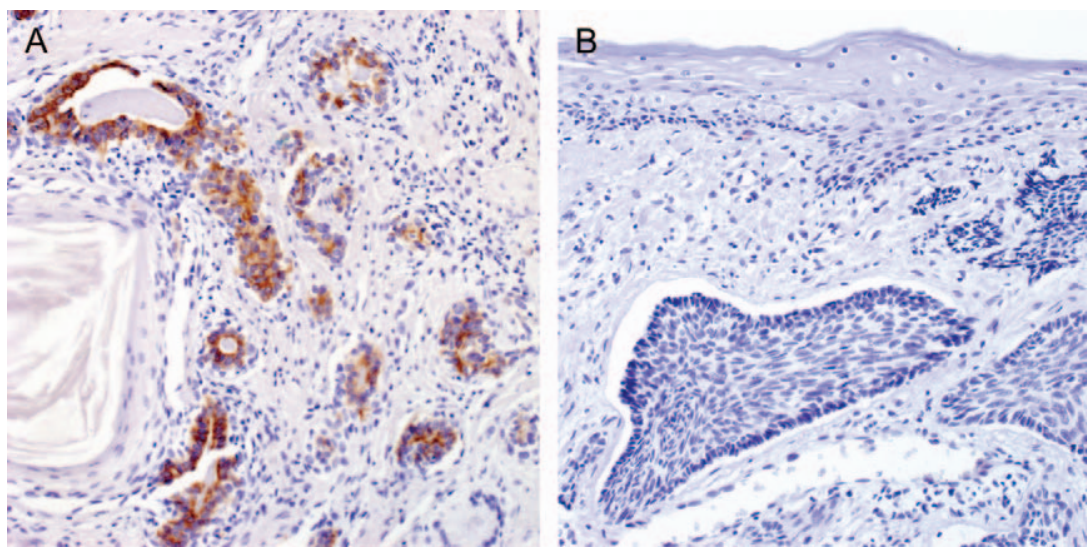

Fig. 3. (A) A proportion of microcystic adnexal carcinoma shows focal strong CD23 positivity in the glandular areas, while morpheaform basal cell carcinoma shows no reactivity for tumor cells $(B)(\times 200)$. 
Fig. 4. (A) CD23 is strongly expressed in mammary Paget's disease, but is negative in pagetoid Bowen's disease $(B)(\times 200)$.
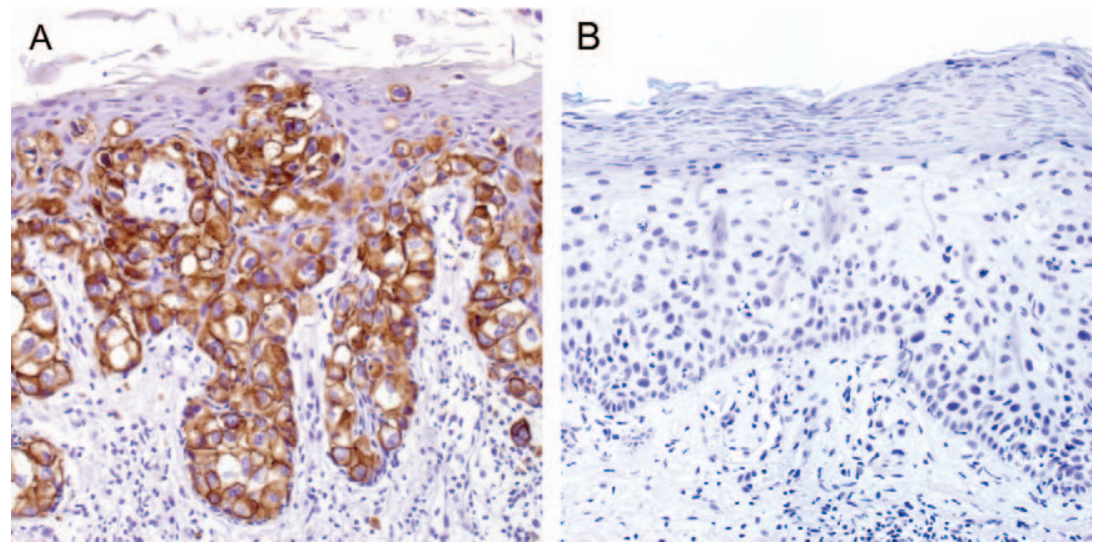

could come from immature poral epithelium. Tumors with apocrine features were focally positive, indicating a possible origin from the deeper portion of the sweat gland.

As MAC is a heterogeneous group of adnexal neoplasm, a variable pattern of staining was observed. As previously reported for CD5, ${ }^{16}$ CD23 also only labeled the glandular elements. The presence of rare CD23-positive cells in some MAC mimickers, such as desmoplastic trichoepithelioma and basal cell carcinoma, is of great interest. According to their morphology and distribution, we suspect that these cells may represent entrapped or pre-existing Merkel cells. Further studies to confirm this hypothesis are necessary.

As expected, because of its derivation from modified apocrine glands, CD23 consistently reacted with the tumor cells of Paget's diseases. These results are compatible with the study by Bogner et al., ${ }^{16}$ in which CD5 labeled Paget's disease, but not other pagetoid lesions. In future, the overall sensitivity and specificity of CD23 needs to be compared with other well-known markers (i.e. cytokeratin $7^{23}$ ) for Paget's disease. The nature and significance of finding occasional staining for epidermal dendrites in melanoma in situ are unknown. It may be labeling the dendritic processes of Langerhans cells, which have been previously reported to express CD23 and therefore assist in antigen presentation. ${ }^{24,25}$

Although CD23 cannot help to distinguish primary cutaneous mucinous eccrine carcinoma from metastatic mucinous carcinoma, the fact that breast and colon adenocarcinoma reacted strongly with CD23 is very interesting. A larger number of cases are needed to confirm our finding. Furthermore, in analogy to CD5, ${ }^{10}$ it would be interesting to investigate possible correlation between the CD23 expression level and the tumor grade of breast carcinomas.

The molecular basis of CD23 expression in nonlymphoid epithelial cells remains unclear. One explanation is that CD23 may react with endogenous $\operatorname{IgE}$ present in the glandular epithelium due to its low binding affinity for IgE. ${ }^{1}$ Alternatively, it may be recognizing an epitope shared by another antigen, such as certain types of cytokeratins. Future studies to determine the expression of other CD23 clones in sweat gland tumors may provide insight into its role in the biology of these tumors.

In conclusion, we found that CD23 is preferentially expressed in apocrine tumors and in a subset of eccrine tumors derived from the eccrine secretory coil. Its expression may help identify the histogenesis
Fig. 5. (A) Mucinous eccrine carcinoma shows diffuse and intense positivity for CD23. (B) Similar staining patterns are detected in breast ductal adenocarcinoma $(\times 200)$.
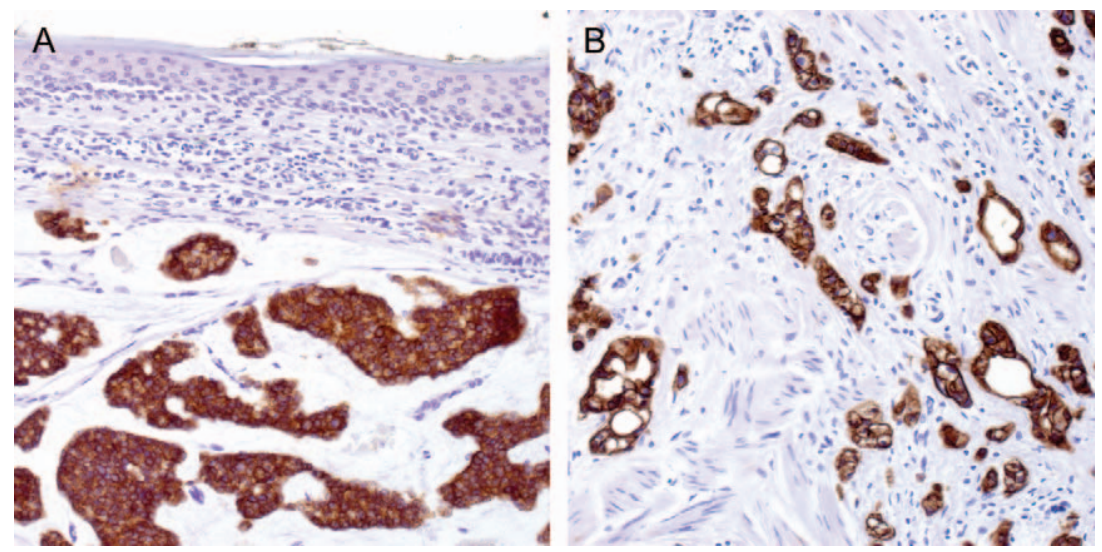


\section{Carvalho et al.}

of sweat gland tumors. CD23 may be of some value in discriminating $\mathrm{MAC}$ from other cutaneous infiltrating tumors. In addition, it may serve as a useful adjunct to the diagnostic immunohistochemical panels for mammary and extramammary Paget's disease.

\section{Acknowledgement}

We thank Mrs Kristina Fields for her excellent technical assistance for CD23 immunohistochemical study.

\section{References}

1. Conrad DH. Fc epsilon RII/CD23: the low affinity receptor for IgE. Annu Rev Immunol 1990; 8: 623.

2. Mossalayi MD, Ouaaz F, Arock M, et al. The role of soluble CD23 on normal and leukaemic myeloid precursor cells. Res Immunol 1992; 143: 439.

3. Fournier S, Rubio M, Delespesse G, et al. Role for low-affinity receptor for $\mathrm{IgE}(\mathrm{CD} 23)$ in normal and leukemic B-cell proliferation. Blood 1994; 84: 1881.

4. Kaiserlian D, Lachaux A, Grosjean I, et al. Intestinal epithelial cells express the CD23/Fc epsilon RII molecule: enhanced expression in enteropathies. Immunology 1993; 80: 90.

5. Thornton CA, Holloway JA, Popplewell EJ, et al. Fetal exposure to intact immunoglobulin $\mathrm{E}$ occurs via the gastrointestinal tract. Clin Exp Allergy 2003; 33: 306.

6. Tu Y, Salim S, Bourgeois J, et al. CD23-mediated IgE transport across human intestinal epithelium: inhibition by blocking sites of translation or binding. Gastroenterology 2005; 129: 928.

7. Kaufmann O, Georgi T, Dietel M. Utility of 123C3 monoclonal antibody against CD56 (NCAM) for the diagnosis of small cell carcinomas on paraffin sections. Hum Pathol 1997; 28: 1373.

8. Langner C, Ratschek M, Rehak P, et al. CD10 is a diagnostic and prognostic marker in renal malignancies. Histopathology 2004; 45: 460.

9. Hishima T, Fukayama M, Fujisawa M, et al. CD5 expression in thymic carcinoma. Am J Pathol 1994; 145: 268.

10. Walsh R, Peston D, Shousha S. Comparison of immunoperoxidase staining of 3 different types of CD5 antibodies in a spectrum of breast lesions. Arch Pathol Lab Med 2001; 125: 781.

11. Kornstein MJ, Rosai J. CD5 labeling of thymic carcinomas and other nonlymphoid neoplasms. Am J Clin Pathol 1998; 109: 722 .
12. Penneys NS. CD44 expression in normal and inflamed skin. J Cutan Pathol 1993; 20: 250.

13. Metze D, Grunert F, Neumaier M, et al. Neoplasms with sweat gland differentiation express various glycoproteins of the carcinoembryonic antigen (CEA) family. J Cutan Pathol 1996; 23: 1 .

14. Ishihara M, Mehregan DR, Hashimoto K, et al. Staining of eccrine and apocrine neoplasms and metastatic adenocarcinoma with IKH-4, a monoclonal antibody specific for the eccrine gland. J Cutan Pathol 1998; 25: 100.

15. Ansai S, Koseki S, Hozumi Y, et al. An immunohistochemical study of lysozyme, CD-15 (Leu M1), and gross cystic disease fluid protein-15 in various skin tumors. Assessment of the specificity and sensitivity of markers of apocrine differentiation. Am J Dermatopathol 1995; 17: 249.

16. Bogner PN, Su LD, Fullen DR. Cluster designation 5 staining of normal and non-lymphoid neoplastic skin. J Cutan Pathol 2005; 32: 50 .

17. Watanabe S, Hirose M, Sato S, et al. Immunohistochemical analysis of cytokeratin expression in eccrine spiradenoma: similarities to the transitional portions between secretory segments and coiled ducts of eccrine glands. Br J Dermatol 1994; 131: 799 .

18. Hernandez FJ. Mixed tumors of the skin of the salivary gland type: a light and electron microscopic study. J Invest Dermatol 1976; 66: 49.

19. Hu CH, Marques AS, Winkelmann RK. Dermal duct tumor: a histochemical and electron microscopic study. Arch Dermatol 1978; 114: 1659.

20. Hashimoto K, DiBella RJ, Borsuk GM, et al. Eruptive hidradenoma and syringoma. Histological, histochemical, and electron microscopic studies. Arch Dermatol 1967; 96: 500.

21. Headington JT. Primary mucinous carcinoma of skin: histochemistry and electron microscopy. Cancer 1977; 39: 1055.

22. Wach F, Hein R, Kuhn A, et al. Immunohistochemical demonstration of myoepithelial cells in sweat gland carcinomas. Br J Dermatol 1994; 130: 432.

23. Battles OE, Page DL, Johnson JE. Cytokeratins, CEA, and mucin histochemistry in the diagnosis and characterization of extramammary Paget's disease. Am J Clin Pathol 1997; 108: 6.

24. Buckley C, Ivison C, Poulter LW, et al. CD23/Fc epsilon R11 expression in contact sensitivity reactions: a comparison between aeroallergen patch test reactions in atopic dermatitis and the nickel patch test reaction in non-atopic individuals. Clin Exp Immunol 1993; 91: 357.

25. Bieber T. Fc epsilon RII/CD23 on epidermal Langerhans' cells. Res Immunol 1992; 143: 445. 